\title{
Unitary Space-Time Modulation via the Cayley Transform
}

\author{
Babak Hassibi and Yindi Jing \\ California Institute of Technology \\ Pasadena, CA 91125 \\ hassibi, yindi@systems. caltech.edu
}

\begin{abstract}
A method of generating good performing USTM constellations using the Cayley transform is proposed. The codes, which can be used for any number of transmit and receive antennas without channel knowledge, are designed based on an information-theoretic criterion, and lend themselves to polynomial-time near-maximum-likelihood decoding using the sphere decoding algorithm.
\end{abstract}

\section{INTRODUCTION}

Consider a wireless communication system with $M$ transmit antennas, $N$ receive antennas and coherent interval $T$. A scheme called USTM that works without channel acknowledge was proposed in [1], in which the transmitted signal $S$ forms a unitary matrix. Further information-theoretic calculations [2] show that at high SNR, USTM can achieve channel capacity. In this paper, we propose to use the Cayley transform to design USTM constellations based on an information-theoretic criterion. The code is not only suitable for any number of $M$ and $N$, but also has polynomial decoding complexity.

\section{CAYley USTM CODES}

The Cayley transform of a complex $T \times T$ matrix $Y$ is defined to be $V=(I+Y)^{-1}(I-Y)$ which provides a oneto-one map from the linear space of skew-Hermitian matrices to the nonlinear Stiefel manifold. So, it is convenient to encode data linearly onto a skew-Hermitian matrix and then apply the Cayley transform to get a unitary matrix. In [3], a class of Cayley codes for differential USTM was proposed. We extend it to the non-square case by choosing our $S$ to be the first $M$ columns of $V$. Let $Y=i A$, by adding structure on the Hermitian matrix $A$ to linearize the near-ML decoder, our USTM codes can be written as $S=\left[\begin{array}{ll}I_{M}+2 B \Delta_{2}^{-1} B^{*} & -2 i \Delta_{2}^{-1} B^{*}\end{array}\right]^{T}$ where $\Delta_{2}=I_{T-M}+B^{*} B-B^{*} A_{11} B+i A_{22}$. $A_{11}=$ $\sum_{q=1}^{Q_{1}} \alpha_{q} A_{11, q}, A_{22}=\sum_{p=1}^{Q_{2}} \beta_{p} A_{22, p}$ are upper-left $M \times M$ and lower-right $(T-M) \times(T-M)$ blocks of $A$. $Q_{1}+Q_{2}=Q$ and $B$ is some fixed $M \times(T-M)$ matrix. $\alpha_{q}$ and $\beta_{p}$ are real scalars chosen from some set $\mathcal{A}$ with cardinality $r$ and carry the information. The $A_{11, q}, A_{22, p}$ are fixed basis matrices. The transmit rate is clearly $(Q / T) \log _{2} r$.

By ignoring the covariance of the additive noise term, the ML decoder can be reduced to some formula that is quadratic in $\left\{\alpha_{q}\right\}$ and $\left\{\beta_{p}\right\}$. Setting the number of unknowns $Q$ less than or equal to the number of independent linear equations [3], which is $2 N(T-M)-N^{2}$ for $T-M \geq N$ and $(T-M)^{2}$ for $T-M<N$, the decoding can be done in roughly $O\left(Q^{3}\right)$ computations by sphere decoding.

From the information-theoretic analysis in $[2,3]$, the optimal distribution for $V$ is an isotropically-random unitary matrix, which implies that $A$ has the matrix Cauchy distribution. Drawing upon the implications from $M=1$ case, we choose the set $\mathcal{A}_{r}$ as the $r$-point discretization of a scalar Cauchy random variable.

Finally, we generalize the approach of [3] and use the criterion $\max _{\left\{A_{11, q}\right\},\left\{A_{22, p}\right\}, B} \mathrm{E} \log \operatorname{det}\left(S^{\perp}-S^{\perp \prime}\right)^{*}\left(S^{\perp}-S^{\perp \prime}\right)$ to choose the basis matrices $A_{11, q}, A_{22, p}$ as well as $B$, where the expectation is over $\alpha_{q}$ and $\beta_{p}$. This optimization may be performed numerically using gradient-ascent methods along with Monte Carlo simulation.

\section{Simulation Results}

Fig. 1 shows that there is little penalty (only around 1db) incurred when using the linearized ML instead of the true ML. In fig. 2 we show that our method gains better performance than training scheme at high SNR even with a higher rate.

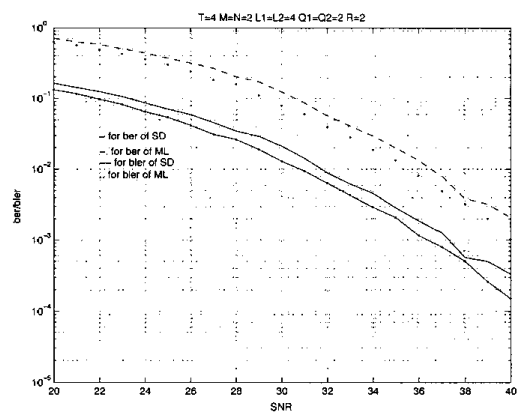

Fig 1: BER and BLER of linearized ML compared to the true ML.

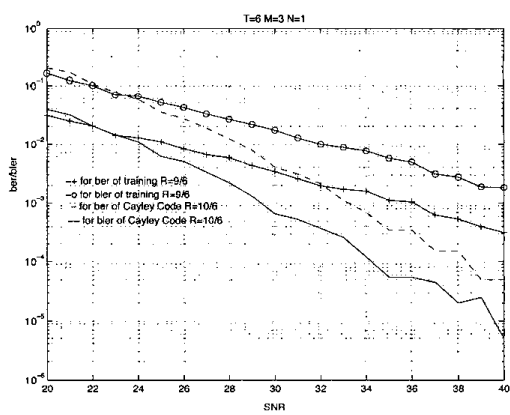

Fig 2: Cayley codes compared to training scheme.

\section{REFERENCES}

[1] B. Hochwald and T. Marzetta, "Unitary space-time modulation for multiple-antenna communication in Rayleigh flat-fading," IEEE Trans. Info. Theory, vol. 46, pp. 543-564, Mar. 2000.

[2] B. Hassibi and T. Marzetta, "Block-fading channels and isotropically-random unitary inputs: The received signal density in closed-form," Submitted to IEEE Trans. on Info. Thy., 2001.

[3] B. Hassibi and B. Hochwald, "Cayley differential unitary spacetime codes," to appear in the IEEE Trans. Info. Theory, 2001. Download available at http://mars.bell-labs.com. 